

\title{
Site- and Regioselectivity of the Hetero-Diels-Alder and Cheletropic Additions of Sulfur Dioxide to a 2,3,6,7-Tetramethylidenebicyclo[3.2.1] octane Derivative
} Brigitte Deguin, Pierre Vogel

\section{- To cite this version:}

Brigitte Deguin, Pierre Vogel. Site- and Regioselectivity of the Hetero-Diels-Alder and Cheletropic Additions of Sulfur Dioxide to a 2,3,6,7-Tetramethylidenebicyclo[3.2.1]octane Derivative. Tetrahedron Letters, 1993, 34 (39), pp.6269-6270. hal-01923579

\author{
HAL Id: hal-01923579 \\ https://hal.science/hal-01923579
}

Submitted on 15 Nov 2018

HAL is a multi-disciplinary open access archive for the deposit and dissemination of scientific research documents, whether they are published or not. The documents may come from teaching and research institutions in France or abroad, or from public or private research centers.
L'archive ouverte pluridisciplinaire HAL, est destinée au dépôt et à la diffusion de documents scientifiques de niveau recherche, publiés ou non, émanant des établissements d'enseignement et de recherche français ou étrangers, des laboratoires publics ou privés. 


\title{
Site- and Regloselectivity of the Hetero-Dlels-Alder and Cheletroplc Additions of Sulfur Dioxide to a 2,3,6,7-Tetramethylldeneblcyclo[3.2.1]octane Derivative.
}

\author{
Brigitte Deguin and Pierre Vogel*
}

Section de Chimie de l'Université de Lausanne, 2, rue de la Barre, CH 1005 Lausanne, Switzerland

\begin{abstract}
Summary: $\mathrm{At}-55^{\circ} \mathrm{C} \mathrm{SO}_{2}$ adds to 4-[(tert-butyl)dimethylsilyloxy]-1-methoxy-2,3,6,7-tetramethylidenebicyclo[3.2.I loctane reversibly and selectively onto the s-cis-butadiene moiety at $\mathrm{C}(2), \mathrm{C}(3)$ giving a single regioisomeric sultine. At $-45^{\circ} \mathrm{C}$ the cheletropic addition of $\mathrm{SO}_{2}$ occurs selectively giving the monosulfolene attached at $C(2), C(3)$.
\end{abstract}

Simple 1,3-dienes such as isoprene and (E)-piperilene undergo hetero-Diels-Alder additions below $-60^{\circ} \mathrm{C}$ and give the corresponding 3,6-dihydro-1,3-oxathiin-2-oxides (sultines) with high regioselectivity. ${ }^{1}$ The sultines are unstable above $-50^{\circ} \mathrm{C}$ and undergo cycloreversion liberating the starting dienes and $\mathrm{SO}_{2}$ that undergo cheletropic additions ${ }^{2}$ above $-40^{\circ} \mathrm{C}$ giving the corresponding sulfolenes. ${ }^{3}$ The Diels-Alder additions of the exocyclic tetraene 1 to strong dienophiles $(X=X)$ such as ethylenetetracarbonitrile, dimethyl acetylenedicarboxylate and maleic anhydride were not site selective and gave mixture of the corresponding monoadducts 2 and 3 together with the bis-adducts $4 .{ }^{4}$ No significant regioselectivity was noticed for the cycloaddition of 1 to methyl propyonate and methyl vinyl ketone. In contrast with these results, we report here that $\mathrm{SO}_{2}$ adds to tetraene 1 with high site-selectivity for both its hetero-Diels-Alder and cheletropic additions, the s-cis-butadiene moiety at $\mathrm{C}(2), \mathrm{C}(3)$ being preferred over that at $\mathrm{C}(6), \mathrm{C}(7)$. Furthermore, in the case of the hetero-Diels-Alder addition $1+\mathrm{SO}_{2}$ only one single regioisomeric sultine was observed.

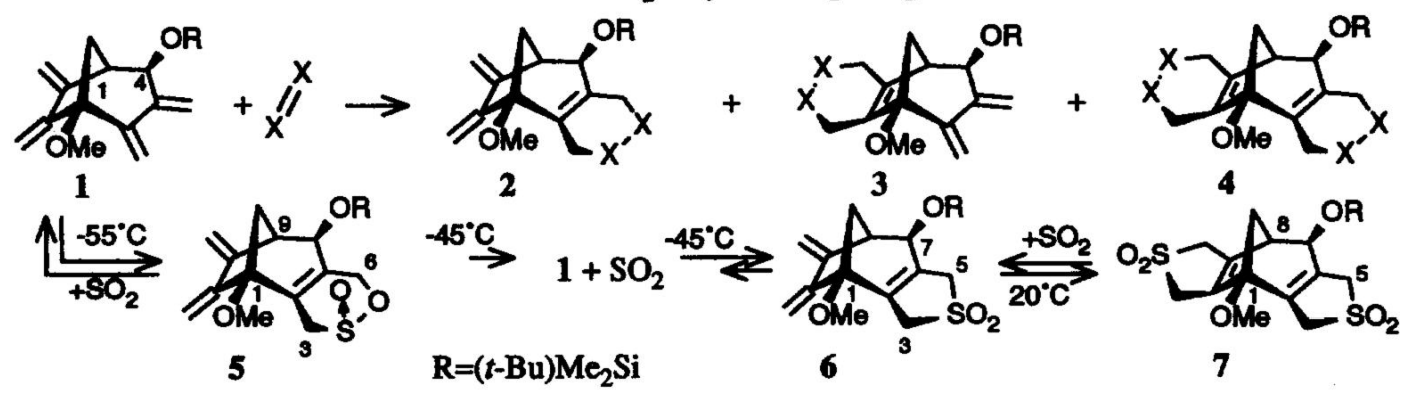

When a $0.2 \mathrm{M}$ solution of 1 in $\mathrm{CD}_{2} \mathrm{Cl}_{2} / \mathrm{SO}_{2}$ 7:1 (w/w) was allowed to stand at $-55^{\circ} \mathrm{C}$ ( $5 \mathrm{~mm}$ sealed NMR tube) the monosultine 5 was formed slowly. After 2 days at $-55^{\circ} \mathrm{C}, 50 \%$ of 5 was formed together with $<5 \%$ of sulfolene 6 and ca. $5 \%$ of an unknown compound the ${ }^{1} \mathrm{H}-\mathrm{NMR}$ spectrum of which was not consistent with an isomeric sultine or sulfolene. After 7 days at $-55^{\circ} \mathrm{C}$, nearly $95 \%$ of 1 had reacted. At $-45^{\circ} \mathrm{C}$, sultine 5 underwent cycloreversion into $1+\mathrm{SO}_{2}$ that reacted slowly to give 6 which was obtained in $85 \%$ yield after $24 \mathrm{~h}$ at $0^{\circ} \mathrm{C}$. The cheletropic addition of a second equivalent of $\mathrm{SO}_{2}$ to give the bis-sulfolene 7 was a very slow process at $0^{\circ} \mathrm{C}$. A good yield of 7 (70-80\%) was obtained when 6 was reacted with a large excess $\mathrm{CD}_{2} \mathrm{Cl}_{2} / \mathrm{SO}_{2} 1: 5(\mathrm{w} / \mathrm{w})$ at $20^{\circ} \mathrm{C}$ for $24 \mathrm{~h}$. After prolonged standing at $0-20^{\circ} \mathrm{C}, 6$ did not equilibrate with an isomeric sulfolene. One cannot exclude yet that both 5 and 6 are preferred for thermodynamic reasons rather then for kinetic reasons. Because 7 decomposed on heating, we could not establish whether 6 
was formed or not by elimination of $\mathrm{SO}_{2}$.

The structures of $5,{ }^{5} 6^{6}$ and $7^{7}$ were deduced from their ${ }^{1} \mathrm{H}$ - and ${ }^{13} \mathrm{C}-\mathrm{NMR}$ spectra and with the help of double irradiation experiments. In the case of 5 the homoallylic coupling constants ${ }^{5} J_{\mathrm{H}, \mathrm{H}}$ between protons $\mathrm{H}-\mathrm{C}(3)$ on one hand, and protons $\mathrm{H}-\mathrm{C}(6)$ and $\mathrm{H}-\mathrm{C}(8)$, on the other hand, ${ }^{8}$ allowed one to distinguish between pseudo-equatorial and pseudo-axial protons at $\mathrm{C}(3)$ and $\mathrm{C}(6)$. Strong NOE's were observed between the proton pairs $\mathrm{H}-\mathrm{C}(8) / \mathrm{H}-\mathrm{C}(9), \mathrm{H}-\mathrm{C}(8) / \mathrm{H}_{\mathrm{e}}-\mathrm{C}(6)$ consistently with the conformation shown for 5 in the Fig. NOE was also observed between the olefinic protons and $\mathrm{H}-\mathrm{C}(9)$ and $\mathrm{MeO}$ protons. The axial $\mathrm{S} O$ moiety of 5 was assumed. ${ }^{9}$

The selectivities reported here for the reaction of 1 with $\mathrm{SO}_{2}$ are striking; they rise a number of questions to which we hope to propose answers when further reactions of $\mathrm{SO}_{2}$ with polyenes analogous to 1 will have been examined.

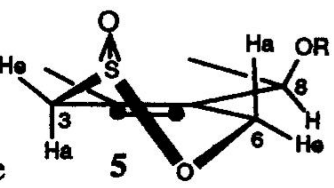

Acknowledgments. We thank $F$. Hoffmann-La Roche \& Co., AG (Basel), the Swiss National Science Foundation, and the Fonds Herbette (Lausanne).

\section{References and Notes}

1. Deguin, B.; Vogel, P. J. Am. Chem Soc. 1992, 114, 9210.

2. Woodward, R. B.; Hoffmann, R. In "The Conservation of Orbital Symmetry", Academic Press, New York, 1970 , pp. 152.

3. De Bruin, G. Proc. K. Ned. Akad. Wet. 1914, 17, 585; Turk, S. D.; Cobb, R. L. In "1,4-Cycloaddition Reactions", Hamer, J., Ed., Academic Press, New York, 1907, pp 13.

4. Burnier, G.; Vogel, P. Helv. Chim. Acta 1990, 73, 985.

5. Data of 5: ${ }^{1} \mathrm{H}-\mathrm{NMR}$ (Bruker, $\left.\mathrm{ARX} 400, \mathrm{CD}_{2} \mathrm{Cl}_{2} / \mathrm{SO}_{2},-55^{\circ} \mathrm{C}\right) \delta_{\mathrm{H}}: 5.5,5.2,5.1,4.8\left(4 \mathrm{~s}, \mathrm{H}_{2} \mathrm{C}=\mathrm{C}(10)\right.$, $\left.\mathrm{H}_{2} \mathrm{C}=\mathrm{C}(11)\right), 4.57$ (ddd, ${ }^{2} J=15,5 J\left(\mathrm{H}_{\mathrm{a}} \mathrm{C}(6), \mathrm{H}_{\mathrm{a}} \mathrm{C}(3)\right)=3,5 J\left(\mathrm{H}_{\mathrm{a}}-\mathrm{C}(6), \mathrm{H}_{\mathrm{e}}-\mathrm{C}(3)\right)=2, \mathrm{H}_{\mathrm{a}}-\mathrm{C}(6)$ ), 4.20 (dd, ${ }^{2} \mathrm{~J}=$ $\left.15,5 J\left(\mathrm{H}_{\mathrm{e}}-\mathrm{C}(6), \mathrm{H}_{\mathrm{a}}-\mathrm{C}(3)\right)=1.0, \mathrm{H}_{\mathrm{e}}-\mathrm{C}(6)\right), 3.82$ (dd, ${ }^{3} \mathrm{~J}=2,{ }^{5} J\left(\mathrm{H}_{\mathrm{a}}-\mathrm{C}(3), \mathrm{H}-\mathrm{C}(8)\right)=1.0, \mathrm{H}-\mathrm{C}(8)$ ), 3.53 (dddd $\left.2 J=17,5^{5} J\left(\mathrm{H}_{\mathrm{a}}-\mathrm{C}(3), \mathrm{H}_{\mathrm{a}}-\mathrm{C}(6)\right)=3,{ }^{5} J\left(\mathrm{H}_{\mathrm{a}}-\mathrm{C}(3), \mathrm{H}_{\mathrm{c}}-\mathrm{C}(6)\right)={ }^{5} J\left(\mathrm{H}_{\mathrm{a}}-\mathrm{C}(3), \mathrm{H}-\mathrm{C}(8)\right)=1.0, \mathrm{H}_{\mathrm{a}}-\mathrm{C}(3)\right), 3.06$ (dd, $\left.{ }^{2} J=17,{ }^{5} J\left(\mathrm{H}_{3}-\mathrm{C}(6), \mathrm{H}_{\mathrm{e}}-\mathrm{C}(3)\right)=2, \mathrm{H}_{\mathrm{e}}-\mathrm{C}(3)\right), 2.85$ (dd, $\left.{ }^{3} J=6,2, \mathrm{H}-\mathrm{C}(9)\right), 2.28\left(\mathrm{~d},{ }^{2} J=10, \mathrm{H}_{s y n}-\mathrm{C}(12)\right), 1.92$ (dd, $\left.{ }^{2} J=10,{ }^{3} J=6, \mathrm{H}_{\text {anti }}-\mathrm{C}(12)\right), 0.90(\mathrm{~s}, \mathrm{t}-\mathrm{Bu}), 0.16 \& 0.15\left(2 \mathrm{~s}, \mathrm{Me}_{2} \mathrm{Si}\right) ;{ }^{13} \mathrm{C}-\mathrm{NMR}\left(100.6 \mathrm{MHz}, \mathrm{CD}_{2} \mathrm{Cl}_{2}\right.$, $\left.-55^{\circ} \mathrm{C}\right) \delta_{\mathrm{C}}: 148.0,145.0(2 \mathrm{~s}, \mathrm{C}(10), \mathrm{C}(11)), 129.4,127.4(2 \mathrm{~s}, \mathrm{C}(2), \mathrm{C}(7)), 109.2,101.0\left(2 \mathrm{t},{ }^{1} \mathrm{~J} \equiv 160\right.$,

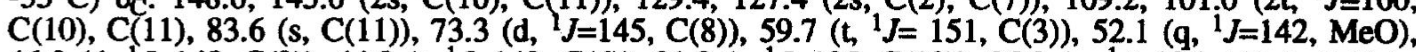
$46.3\left(\mathrm{~d},{ }^{1} J=140, C(9)\right), 44.8\left(\mathrm{t},{ }^{1} J=140, C(6)\right), 31.3\left(\mathrm{t},{ }^{1} J=135, C(12)\right), 25.3\left(\mathrm{q},{ }^{1} J=125, \mathrm{t}-\mathrm{Bu}\right), 17.7(\mathrm{~s}$,



6. Data of 6: ${ }^{1} \mathrm{H}-\mathrm{NMR}\left(360 \mathrm{MHz}, \mathrm{CD}_{2} \mathrm{Cl}_{2} / \mathrm{SO}_{2}, 0^{\circ} \mathrm{C}\right) \delta_{\mathrm{H}}: 5.62,5.38,5.22,4.95(4 \mathrm{~s}), 4.0-3.9\left(\mathrm{~m}, \mathrm{H}_{\mathrm{a}}-\mathrm{C}(3)\right.$, $\left.\mathrm{H}_{\mathrm{a}}-\mathrm{C}(5), \mathrm{H}-\mathrm{C}(7)\right), 3.8-3.72$ \& 3.62-3.60 (2m, $\left.\mathrm{H}_{\mathrm{b}}-\mathrm{C}(3), \mathrm{H}_{\mathrm{b}}-\mathrm{C}(5)\right), 3.33(\mathrm{~s}, \mathrm{MeO}), 2.98(\mathrm{~m}, \mathrm{H}-\mathrm{C}(8)), 2.22$ $\left(\mathrm{d},{ }^{2} J=10\right) \& 1.93\left(\mathrm{dd},{ }^{2} J=10,{ }^{3} J=6, \mathrm{H}_{2} \mathrm{C}(11)\right), 0.95(\mathrm{~s}, \mathrm{t}-\mathrm{Bu}), 0.20$ \& $0.15\left(2 \mathrm{~s}, \mathrm{Me}_{2} \mathrm{Si}\right) ;{ }^{13} \mathrm{C}-\mathrm{NMR}$ $\left(100.6 \mathrm{MHz}, \mathrm{CD}_{2} \mathrm{Cl}_{2} / \mathrm{SO}_{2}, 0^{\circ} \mathrm{C}\right) \delta_{\mathrm{C}}: 147.4,145.3,139.9,128.6(4 \mathrm{~s}), 109.7\left(\mathrm{t}, 2 \mathrm{C},{ }^{1} \mathrm{~J}=159\right), 84.3(\mathrm{~s}$, $\mathrm{C}(1)), 72.4\left(\mathrm{~d},{ }^{1} J=146, \mathrm{C}(7)\right), 58.8\left(\mathrm{t},{ }^{1} J=143, \mathrm{C}(3)\right), 55.7\left(\mathrm{t},{ }^{1} J=157, \mathrm{C}(5)\right), 52.6\left(\mathrm{q},{ }^{1} \mathrm{~J}=143, \mathrm{MeO}\right)$, $47.4\left(\mathrm{~d},{ }_{J=141}, \mathrm{C}(8)\right), 31.8\left(\mathrm{t},{ }_{J=135, C}(11)\right), 25.8\left(\mathrm{q},{ }_{J=125)}, 18.3(\mathrm{~s}),-4.61 \&-4.74\left(2 \mathrm{q},{ }^{1} \mathrm{~J}=119\right)\right.$.

7. Data of 7: ${ }^{1} \mathrm{H}-\mathrm{NMR}\left(360 \mathrm{MHz}, \mathrm{SO}_{2} / \mathrm{CD}_{2} \mathrm{Cl}_{2}, 20^{\circ} \mathrm{C}\right) \delta_{\mathrm{H}}: 4.1(\mathrm{~m}, \mathrm{H}-\mathrm{C}(7)), 4.0-3.75\left(\mathrm{~m}, 8 \mathrm{H}, \mathrm{H}_{2} \mathrm{C}(3)\right.$, $\left.\mathrm{H}_{2} \mathrm{C}(5), \mathrm{H}_{2} \mathrm{C}(10), \mathrm{H}_{2} \mathrm{C}(12)\right), 3.35(\mathrm{~s}, \mathrm{MeO}), 3.08(\mathrm{~m}, \mathrm{H}-\mathrm{H}(8)), 2.58(\mathrm{dd}, 2 J=10,3 \mathrm{~J}=5) \& 2.45(\mathrm{~d}, 2 \mathrm{~J}=$ $\left.10, \mathrm{H}_{2} \mathrm{C}(14)\right), 0.90(\mathrm{~s}, \mathrm{t}-\mathrm{Bu}), 0.16,0.15\left(2 \mathrm{~s}, \mathrm{Me} \mathrm{Si}\right.$; $\left.{ }^{13} \mathrm{C}-\mathrm{NMR}\left(100.6 \mathrm{MHz}, \mathrm{SO}_{2} / \mathrm{CD}_{2} \mathrm{Cl}_{2}\right), 20^{\circ} \mathrm{C}\right) \delta_{\mathrm{C}}:$ 125.5 (s, C(2), C(4)), 115.6, 113.9 (2s, C(6), C(9)), 70.9 (s, C(1)), 65.3 (d, $J=143, \mathrm{C}(7)$ ), 58.3, 57.0, $56.8,56.1\left(4 t,{ }^{1} J=146\right), 52.9\left(q,{ }^{1} J=143, M e O\right), 47.2\left(d,{ }^{1} J=144, C(8)\right), 40.8\left(t,{ }^{1} J=138, C(14)\right), 26.1(q$, $1_{J=125),} 18.7(\mathrm{~s}),-4.5,-4.6(2 \mathrm{q})$.

8. Barfield, M.; Sternhell, S. J. J. Am. Chem. Soc. 1972, 94, 1905; Mahaim, C.; Carrupt, P.-A.; Vogel, P. Helv. Chim. Acta 1985, 68, 2182.

9. Juaristi, E.; Cuevas, G. Tetrahedron 1992, 48, 5019 and references cited therein. 